In any event, the large difference between the valence frequencies of the excited states shows that one of them is relatively much more anti-bonding than the other.

The detailed discussion of the very important scheme of levels given by Mulliken must be postponed until new experimental data in the $2000 \mathrm{~A}$. region are available. We are now engaged in such experiments. $\mathrm{SeO}_{2}$.-The $\mathrm{C}$ system extending between 5000 and $3400 \mathrm{~A}$. has been photographed at temperatures ranging from $300^{\circ}$ to $500^{\circ} \mathrm{C}$., the pressure varying from $50 \mathrm{~mm}$. to $450 \mathrm{~mm}$. mercury, with absorbing layers of $2-400 \mathrm{~cm}$. Our measurements are generally in accordance with those of Evans ${ }^{3}$ and Choong Shin-Piaw ${ }^{3}$, who give no interpretation of their results.

The most characteristic feature of the system is the presence of several $\nu_{2}^{\prime}$ series with $\nu_{2}^{\prime} \cong 200 \mathrm{~cm}$. $^{-1}$. For the normal state we obtain $\boldsymbol{v}_{1}{ }^{\prime \prime}=910 \leqslant \mathrm{~cm}^{-1}$, conflrming our previous result based on the analysis excited valence frequency indicates that valence bonds are practically unchanged.

In the long wave-length region of the spectrum, the bands are degraded towards the red, and the reverse is observed on the ultraviolet side; whereas in the centre, the bands are diffuse. This fact seems to be due to an isotope effect.

In any event, the noticeable intensity of the system and the absence of antisymmetrical frequencies show that it results from an allowed transition. It may correspond with the $C$ system of $\mathrm{SO}_{2}\left(4 a_{1} \rightarrow 2 b_{1}\right)$. Metropolis ${ }^{8}$ noted that the ratio $I_{c} / I_{b}$ is practically constant $(1 \cdot 17)$ for a series of angular molecules such as $\mathrm{SO}_{2}, \mathrm{NO}_{2}, \mathrm{ClO}_{2}$. If we extend the validity of this rule to $\mathrm{SeO}_{2}$, it provides an apex angle of about $130^{\circ}$. Furthermore, we have noted that the ratio of the antisym$130^{\circ}$. Furthermore, we have noted that the ratio of the antisymconstant with the value 1.17 (the identity of the constants seems to be fortuitous).

On the basis of this relation, we obtain for $\mathrm{SeO}$, the value $v_{3}^{\prime \prime}=$ On the basis of this relation, we obtain for $\mathrm{SeO}_{2}$ the value $\nu_{3}{ }^{\prime \prime}=$
$1050 \mathrm{~cm} .^{-1}$. On the other hand, the general character of the spectrum $1050 \mathrm{~cm} .^{-1}$. On the other hand, the general
indicates that $v_{3}{ }^{\prime \prime}$ must be about $400 \mathrm{~cm} .^{-1}$.

With the three fundamental frequencies obtained, and by using With the three fundamental frequencies obtained, and by using a valence-deformation force fleld, we compute an apex angle of about
$160^{\circ}$. In order to obtain a more plausible angle of $130^{\circ}$, it is sufficient $160^{\circ}$. In order to obtain a more plausible angle of $130^{\circ}$, it is sufficient $0.5 \times 10^{5} \mathrm{dyne} / \mathrm{cm}$. Hence we seem to have a new case of an angular $0.5 \times 10^{5}$ dyne $/ \mathrm{cm}$. Hence we seem to have a new case of an angular

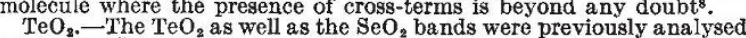
$\mathrm{TeO}_{2}$. - The $\mathrm{TeO}_{2}$ as well as the $\mathrm{SeO}_{2}$ bands were previously analysed
by Choong Shin $\mathrm{Piaw}^{3}$, who divided the spectrum into several electronic by Choong Shin Piaw ${ }^{3}$, who divided the spectrum into several electronic
systems. As important objections can be made to his vibrational systems. As important objections can be made to his vibrational we have undertaken a new investigation of the main $b$ system situated we have undertaken a new investigation of the main $b$ system situated between 3000 and $4500 \mathrm{~A}$. with a maximum absorption at $3600 \mathrm{~A}$. Our measurements are in satisfactory agreement with those of
Choong, but we have been able to correlate all the bands of the system with only one valence symmetrical frequency in each state : $v_{1}^{\prime \prime}=815 \mathrm{~cm}^{-1} ; v_{1}^{\prime}=650 \mathrm{~cm}^{-1}$.

According to this result, the system may be considered as analogous to the $b$ system of $\mathrm{SeO}_{2}$. In addition to the main bands, some fainter bands situated on the short wave-length side of the spectrum can be arranged in several series with $\nu \cong 200 \mathrm{~cm}^{-1}$. It can be identifled with a deformation frequency in the upper level, but we cannot yet decide whether it belongs to the same electronic transition.

It is perhaps worth while to note the close agreement between the fundamental valence frequency of these three molecules $(1154,910$, $815 \mathrm{~cm}^{-1}$ ) and the fundamental frequency of the corresponding diatomic molecule $\left(1123,908,796 \mathrm{~cm}^{-1}\right)$.

Details of our investigation will be published elsewhere in the near future.

Department of Physical Chemistry,

JULES DUCHESNE

\section{Institute of Astrophysics,}

B. ROSEN

University of Liege.

March 20.

' Schomaker, V., and Stevenson, D. P., J. Amer. Chem. Soc., 62, 1270

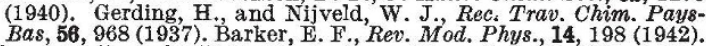
${ }^{2}$ Palmer, K. J., and Elliott, N., J. Amer. Chem. Soc., 60, 1389 (1938) ${ }^{3}$ Henri, v., Nature, 125, 275 (1930). Evans, S. F., Nature, 125, 528 (1930). Clements, J. H., Phys. Rev., 47, 224 (1935). Price, W. C., and Simpson, D. M., Proc. Roy. Soc., A, 165, 272 (1938). Choong Shin-Piaw, C.R. Acad. Sci. Paris, 2J2, 127 (1936); 208, 239 (1936) 4 Metropolis, N., Phys. Rev., 60, 295 (1941). Metropolis, N., and Beutler, H., Phys. Rev., 57, 1078 (1940).

${ }^{5}$ Duchesne, J., and Rosen, B., Physica, 8, 540 (1941)

${ }^{6}$ Metropolis, N., Phys. Rev., 60, 283 (1941).

? Mulliken, R. S. Rev, Mod. Phys., 14, 204 (1942).

- Duchesne, J., Mém. Soc. Roy. Sci. Liège, 1, fasc. 2, 429 (1943).

\section{The Normal and Power Vortex in Meteorology}

NUMERous attempts ${ }^{1}$ have been made to apply the simple plane vortex of hydrodynamical theory to the structure of symmetrica pressure centres on the synoptic weather chart. These studies, which are based on the so-called simple vortex, in which the tangentia velocity at a distance $r$ from the centre is given by

$$
v r=a \text { constant, }
$$

are inadequate, since the simple vortex encounters convergence difficulties both at $r=0$ and as $r \rightarrow \infty$. Such a system of winds would have an infinite kinetic energy associated with it, while a consistent pressure fleld would have an infinite potential energy.

A study has been made of plane vortex systems which are free from these embarrassing disadvantages, in particular the 'normal' vortex, in which the pressure fleld is given by

$$
p=\text { const. }+h \exp -r^{2} / a^{2},
$$

and the power vortex, the fleld for which is

$$
p=\text { const. }+h /\left(1+r^{2} / a^{2}\right)^{1}+\mu \text {. }
$$

The normal vortex is completely defined by two parameters, the strength, $h$, and the spread or dispersion, $a$. The power vortex dis. tribution resembles the normal distribution in general proflle but the pressure decreases more slowly with $r$ in the outer part of the vortex. It requires for its complete speciflcation the index $\mu$ in addition to the two parameters already mentioned.

In applying such vortices to the large pressure systems of the synoptic weather chart, it is assumed that the variation of surface density and of the earth's vertical component of rotation does not vary within the vortex. The retarding influence of surface friction on wind speed is also ignored.

For the cyclone or anti-cyclone of temperate latitudes, it is assumed that winds are nearly geostrophic. With this assumption it can be shown that

$V_{m} R / h=$ a constant,

where $V_{m}$ is the maximum wind speed at distance $R$ from the centre. This constant is equal to $4.8 \times 10^{6} \mathrm{gm}^{-1} \mathrm{~cm} .^{3} \mathrm{sec}$. in the case of a normal distribution, and to $2 \cdot 9 \times 10^{5}$ in the case of the power vortex, index zero. The mean value for this parameter determined from data for seventeen cyclones published by Goldie $^{2}$ is $2.5 \times 10^{8}$.

The power vortex gives rather a better fit than the normal vortex, wind speeds in which fall off too rapidly with distance from the centre. It can be shown that the potential energy associated with a normal vortex is

$\pi a^{2} h Z C_{p} / 2\left(C_{p}-C_{v}\right)$,

where $C_{p}$ and $C_{v}$ are the specific heats of air at constant pressure and constant volume, respectively, and $Z$ is the vertical extent of of the vortex is

$\left.\pi Z h^{2} / 18 \rho_{0} \omega\right)^{2}$, where $\omega^{2}$ is the vertical component of the earth's rotation, and $\rho_{0}$ the
density of surface air. Analogous expressions hold in the case of the power vortex.

A cyclone of fixed strength loses kinetic energy as it moves to higher latitudes. This may be compensated by a decreasing spread leading to a diminution in the (negative) potential energy of the vortex. The precise interchange of energy in the atmosphere can only be The precise interchange of energy in the atmosphere can only be vortex, or the power vortex, provides models of the cyclone or anticyclone, by means of which one is able to discuss the large-scale cyclone, by means of which one in temperate regions the wind is approximately geostrophic, but in the tropics the geostrophic control is weaker, and in the inner parts of the tropical cyclone, at least, the

one balancing the pressure gradient. A vortex in which the cyclostrophic control is predominant is
rather different from that we have already discussed. The constant of such a system is

$V_{m}^{2} / h$.

This constant in the case of a normal vortex is $600 \mathrm{gm}^{-1} \mathrm{~cm}^{3}$, or for a power vortex, index $1,420 \mathrm{gm}^{-1} \mathrm{~cm}^{3}$. The mean value of $V_{m}^{2} / h$ for eighteen tropical cyclones is $490 \mathrm{gm}^{-1} \mathrm{~cm}^{3}$, with extreme values of 190 and 910 . If allowance is made for the effects of surface friction, the value of this ratio will be increased to more than 700

The normal yortex would seem to ft the data better than the power vortex for small $r$, but for moderate values of $r, \boldsymbol{V}$ falls off too rapidly

The study of normal and power vortices has so far been confined to large-scale atmospheric systems; but there is the likelihood of applications being found to vortices of the scale encountered in connexion with turbulence phenomena, and, indeed, in all hydrodynamical
studies in which the overall energy of individual vortices must be

A more complete account will be published elsewhere

I am indebted to the Director of the Meteorological Office for permission to communicate the above.

R. W. JAMES

Meteorological Office,

6 Drumsheugh Gardens,

Edinburgh, 3.
March 14.

${ }^{1}$ Goldie, A. H. R. "Kinematical Features of Depressions", M.O. Geophys. Mem. No. 72 (1937), gives full references to the litera ture.

${ }^{2}$ Goldie, A. H. R., "Depressions as Vortices", M.O. Geophys. Mem. No. 79 (1939)

\section{Linear 'Curves of Best Fit'}

THE text-book treatment of linear curves of best flt purports to give the best estimate of the relation $w_{1}=\rho v_{1}$ between variables of true value $w_{1}, v_{1}$, from a 3 et of measured values, $w, v$. It is a pplicable when only one parameter is subject to errors of measurement. Thus, when only the values of $w$ are subject to error, the best estimate is given by the curve which minimizes the sum of squares of deviation of $\omega$, that is, the regression line of $w$ on $v$; and for errors in $v$ only,

We have had occasion to ask the question, What is the best estimate of $\rho$ when both $w$ and $v$ are subject to normal errors? The following rather intuitive solution has been verifled rigorously for the special case of a sample containing a normal distribution of true values. It is of wide application and certain of its implications are of some

The standard deviations $\sigma_{w}, \sigma_{v}$ of the measured values are assumed to be constant throughout the range, and $w, v$ are taken relative to 